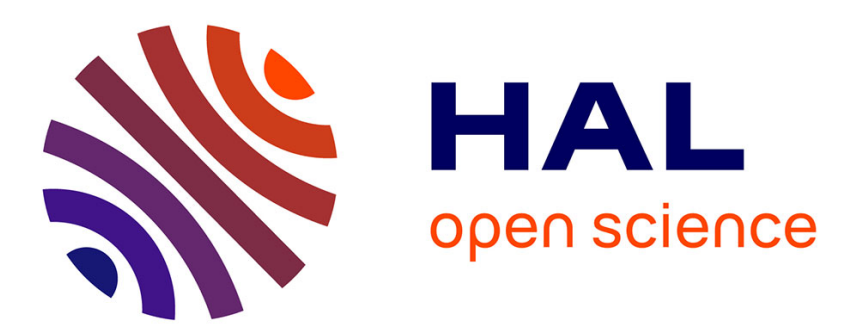

\title{
Discrete and continuum modelling of size effects in architectured unstable metamaterials
}

\author{
Claudio Findeisen, Samuel Forest, Jörg Hohe, Peter Gumbsch
}

\section{To cite this version:}

Claudio Findeisen, Samuel Forest, Jörg Hohe, Peter Gumbsch. Discrete and continuum modelling of size effects in architectured unstable metamaterials. Continuum Mechanics and Thermodynamics, 2020, 10.1007/s00161-020-00870-8 . hal-02488079

\section{HAL Id: hal-02488079 \\ https://hal.science/hal-02488079}

Submitted on 16 Oct 2021

HAL is a multi-disciplinary open access archive for the deposit and dissemination of scientific research documents, whether they are published or not. The documents may come from teaching and research institutions in France or abroad, or from public or private research centers.
L'archive ouverte pluridisciplinaire HAL, est destinée au dépôt et à la diffusion de documents scientifiques de niveau recherche, publiés ou non, émanant des établissements d'enseignement et de recherche français ou étrangers, des laboratoires publics ou privés. 


\title{
Discrete and continuum modelling of size effects in architectured unstable metamaterials
}

Received: 24 June 2019 / Accepted: 28 January 2020

(C) Springer-Verlag GmbH Germany, part of Springer Nature 2020

\begin{abstract}
Metamaterials made of bi-stable building blocks gain their promising effective properties from a micromechanical mechanism, namely buckling, rather than by the chemical composition of its constituent. Both discrete and continuum modelling of unstable metamaterials is a challenging task. It requires great care in the stability analysis and a kinematic enhanced continuum theory to adequately describe the softening behaviour and related size effects. This paper presents a detailed analytical and numerical investigation of the modelling capabilities of a gradient enhanced continuum model compared to a discrete modelling approach which has been proven to be qualitatively consistent with experimental results on small structures. It is demonstrated that the gradient model is capable of describing size effects with respect to stability of small structures; however, the limit of very large structures is found to be inconsistent with both the discrete model and the Maxwell rule of a classical continuum. Based on an analytical investigation of the size of the energy barrier between local minima, it is discussed how the consistency of the limit case of both discrete and continuum models can be restored by redefining the classical meaning of stability.
\end{abstract}

Keywords Gradient continuum $\cdot$ Stability size effects $\cdot$ Unstable metamaterials $\cdot$ Softening and localization

\section{Introduction}

One of the fundamental assumptions of classical continuum mechanics is the assumption of a continuous distribution of particles [1]. If this assumption fails, the kinematic description can be enhanced in the framework of higher-order or higher-grade continua. Well-known examples of such extended theories are the Cosserat continuum [2], the micromorphic- [1] or second gradient continua [3], and its various special cases (e.g. [4] or [5]). Extended continuum theories become necessary if microstructural detail is relevant for the effective material response. This is for example the case in the continuum modelling of (boundary) size effects [6], or wave dispersion [7]. Moreover, the microstructural aspect is also relevant in the description of localization and material failure phenomena. Both material failure and localization are driven by the microstructure, and so it appears natural that their accurate description also requires a kinematic enhancement. A kinematic enhancement

Communicated by Andreas Öchsner.

C. Findeisen · P. Gumbsch

Institute for Applied Materials, Karlsruhe Institute of Technology (KIT), 76128 Karlsruhe, Germany

C. Findeisen $(\varangle) \cdot$ J. Hohe $\cdot$ P. Gumbsch

Fraunhofer Institute for Mechanics of Materials IWM, 79108 Freiburg, Germany

E-mail: Claudio.Findiesen@iwm.fraunhofer.de

S. Forest

Centre des Matériaux, Mines-ParisTech, CNRS UMR 7633, PSL Research University, BP 87, 91003 Evry Cedex, France 
for localization and failure especially becomes relevant to prevent mesh dependency in the numerical analysis of failure [8-11].

More recently, related problems arise by modelling so-called metamaterials. Metamaterials gain their unusual and extreme effective properties mainly from micromechanical mechanisms rather than from the chemistry of their constituent materials. It is thus not surprising that their "homogenization" also requires extended continuum models. Examples are the description of wave dispersion and band gaps by kinematically enhanced models $[12,13]$, or the description of a tension-twist coupling by a Cosserat continuum as they are observed in chiral lattices [14]. Pushing this to the limit, one can design microstructures that can only be described within continuum mechanics if one includes higher gradients of second, third or even 4th order [15].

In this paper, we focus on the continuum description of unstable metamaterials as they have been recently presented and analysed [16,17]. The energy dissipation within these microstructures is driven by the sudden state transformation which enables a separation of timescales between external loading and viscous dissipation in the constituent material. This in turn leads to a non-viscous effective material response with dissipation in the static limit. Moreover, the in-depth analysis of these metamaterials has revealed pronounced stability and hysteresis size effects [17].

The effective material behaviour of composites or microstructured materials is often obtained by homogenization based on the Hill-Mandel framework, a technique well established for classical continuum theories [18]. However, an extension of the Hill-Mandel homogenization framework towards generalized continua is not so well established and its application is inhibited by the strong assumptions that have to be made according to the deformation of the microstructure. Two possibilities to extend the classical Hill-Mandel homogenization are presented and discussed in the literature. The first approach [19] assumes a polynomial microdeformation which cannot be relaxed using a periodic microfluctuation as often done in the classical homogenization framework. The second approach [20,21] applies Lagrange multipliers and thus assumes that the true microdeformation can be mimicked by applying a polynomial volume force onto the unit cell. Furthermore, a promising computational homogenization approach has been presented in [22]. In there, the two-scale numerical simulation of a periodically buckling microstructure is performed by enhancing the macroscopic displacement field by a buckling mode modulation variable $v_{1}(\mathbf{X})$ which is used to smoothly vary the periodic buckling pattern that has to be known a priori. This method, however, requires that the buckling pattern is periodic such that a periodic cell can be used for the homogenization. Compared with this, the unstable microstructure that motivates this work is characterized by an instability that leads to a localization within a single cell and hence a periodicity length of infinity.

We directly investigate the modelling capabilities of a gradient enhanced material model without homogenization. The focus is on a one-dimensional strain gradient enhanced model with a strain energy density that is quadratic in the gradient part and non-convex with respect to strain. This modelling approach will be compared to results obtained from a discrete model which has been proven to be qualitatively consistent with experimental results $[16,17]$. Previous results on similar elastic discrete and gradient enhanced models with non-convex potentials include: [23] a rigorous investigation of a discrete local model. The focus there is on the role of the unstable region of the single element for the stability and the possible transformation processes in a finite chain of unstable elements for both the Dirichlet and the von Neumann problem. Limited to the von Neumann problem, this work is extended in [24] investigating the role of finite fluctuations for the possible transformation processes. The origin of the so-called nucleation peak, experimentally observed in the phase transformation of metals and predicted by both non-local discrete and non-local continuum models, is intensively analysed in [25]. Finally, [26] analyses discrete models with non-local interactions and their mathematical relation to gradient enhanced continuum models. Beside the aforementioned elastic models, gradient plasticity models have been frequently applied to regularize compaction [8-10] and shear band instabilities [11] in metals as well as to predict size effects in metal plasticity [27] or in microstructured materials like foams [28]. However, an investigation of gradient enhanced continuum models with respect to localizing unstable metamaterials is still lacking. The application to unstable metamaterials is particularly interesting since it is unclear to which extent a continuum model is capable of describing the exotic behaviour of such structures and related size effects.

More precisely, we investigate the continuum description of size effects in two limit cases: The upper limit where the number of unstable cells is increased to infinity $(N \rightarrow \infty)$, and the lower limit of only a few unstable cells $(N=1,2,3,4, \ldots)$. In the upper limit $(N \rightarrow \infty)$ the inconsistency of both the discrete and the gradient model to the so-called Maxwell rule of equal area [29] will be discussed. It is shown that the enhanced continuum model is consistent with the discrete model only if one includes finite fluctuations in both models. 


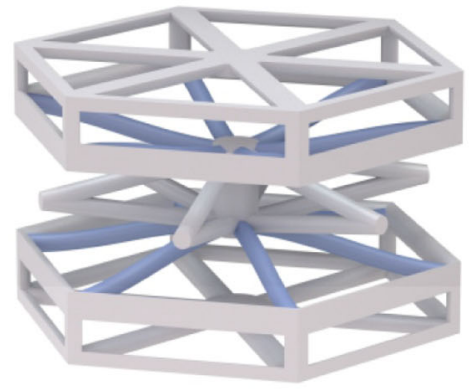

(a)

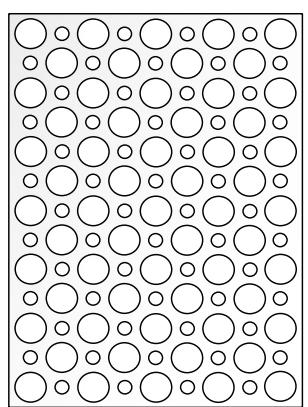

(b)

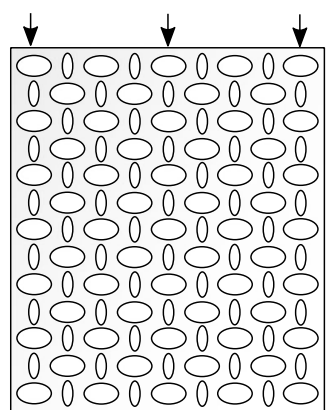

(c)

Fig. 1 Different microstructural designs for unstable metamaterials. a Hexagonal unit cell with cosine-shaped buckling elements (blue) which buckle upon vertical compression [16] and $\mathbf{b}, \mathbf{c}$ biholar sheet as presented in [30] that exhibit a pattern transformation from circular holes (a) to elliptical holes with different orientations (b). (Figure (a) with permission from [16] Figures (b) and (c) are reproduced from [30])

For the lower limit of only a few cells, it is demonstrated that stability size effects can be described by a second gradient theory.

\section{Unstable metamaterials-microstructures and main mechanism}

Unstable metamaterials gain their unusual effective properties from a unit cell that shows a bi-stable stressstrain (or force displacement) behaviour as schematically shown in Fig. 2 and discussed in more detail later.

The microstructure (or unit cell) that has motivated the investigation in this paper is shown in Fig. 1a. The bi-stability within this structure is induced by the buckling of the cosine-shaped buckling elements upon a compression in the vertical direction. In a lattice of many of such cells, buckling appears suddenly with a corresponding strain energy release leading to local oscillations of the microstructure. These oscillations are damped - and thus, the corresponding energy is dissipated to heat — by the viscosity of the constituent material. Importantly, the timescale of this dissipation process is mainly determined by the buckling process and thus by the design of the microstructure. For a static loading or a loading with frequency much smaller than the eigenfrequency of the oscillations initiated by the buckling, this leads to an overall energy dissipation which is in a certain range independent of the timescale of external loading even though the constituent material behaves viscous. At the same time, the microstructure is designed such that it also shows a fully reversible deformation upon unloading. The latter property, i.e. the reversibility, clearly differentiates this class of materials from usual metals where energy dissipation is related to plastic deformation. Furthermore, the bi-stability allows the design of so-called mechanically programmable materials where the effective behaviour for the same strain or stress state can be different due to a different loading history. All of the aforementioned properties have been realized experimentally in [16] and analysed analytically and numerically in [17].

Clearly, the microstructure depicted in Fig. 1a is not the only possibility to realize a bi-stable stress-strain behaviour. As a further example, the microstructure presented in [30] is represented in Fig. 1b, c; the bistability is here obtained from a pattern transformation leading to the formation of an alternating arrangement of ellipsoidal holes with different orientations. Beside these two somehow different microstructural approaches, several other alternative designs have been presented that result in similar effective properties (e.g. [31,32]).

\section{Analytical investigations-the discrete model}

The problem of interest is here the behaviour of an array (or chain) of $N$ unit cells (or springs) with a non-convex free energy density $w(\varepsilon)$ as the simplest one-dimensional discrete model for the microstructure of unstable metamaterials. The focus will be on the displacement boundary condition where the structure is fixed at one end $\left(u_{0}=0\right)$ and a displacement $U$ is applied at the other end $\left(u_{N}=U\right)$. Stable equilibrium configurations $\varepsilon_{0}$ are given by local minimizers of the total elastic energy 


$$
W=\frac{L}{N} \sum_{i=1}^{N} w\left(\varepsilon_{i}\right)+L \bar{\sigma}\left(\bar{\varepsilon}-\frac{1}{N} \sum_{i}^{N} \varepsilon_{i}\right) .
$$

The first part of this equation accounts for the elastic energy of the individual unit cells and the second part utilizes a Lagrange multiplier $\bar{\sigma}$ (i.e. external force) to account for the boundary condition; furthermore, the length of an individual unit cell is chosen as $s=L / N$, such that $N$ unit cells give the overall length $L$, and $\varepsilon_{i}$ and $\bar{\varepsilon}$ are the strain of cell $i$ and the strain of the overall structure respectively. Necessary condition for a minimizer of (1) is that the first derivatives with respect to the strains and with respect to the Lagrange multiplier vanish

$$
\begin{aligned}
\partial_{i} w\left(\varepsilon_{i}\right) & =\bar{\sigma} \quad \forall i=1, \ldots, N, \\
\frac{1}{N} \sum_{i}^{N} \varepsilon_{i} & =\bar{\varepsilon} .
\end{aligned}
$$

The condition for stability is nothing but the sufficient condition for a local minimizer: An equilibrium configuration is said to be stable if the change in the overall potential $\mathrm{d} W$ due to a small perturbation $\mathrm{d} \varepsilon$ is positive. From the Taylor series expansion around an equilibrium state $\boldsymbol{\varepsilon}_{0}$

$$
\mathrm{d} W=W\left(\boldsymbol{\varepsilon}_{0}\right)+\partial_{i} W\left(\boldsymbol{\varepsilon}_{0}\right) \mathrm{d} \varepsilon_{i}+\partial_{i j} W\left(\boldsymbol{\varepsilon}_{0}\right) \mathrm{d} \varepsilon_{i} \mathrm{~d} \varepsilon_{j}+\mathcal{O}\left(|\mathrm{d} \boldsymbol{\varepsilon}|^{3}\right),
$$

it can be concluded that for sufficient small perturbations $\mathrm{d} \boldsymbol{\varepsilon}$ a stable equilibrium configuration is equivalent to a positive definite second derivative

$$
\varepsilon_{i} \partial_{i j} W \varepsilon_{j} \geq 0 \quad \forall \boldsymbol{\varepsilon} \in \mathcal{Z},
$$

where $\mathcal{Z}$ is the space of admissible deformations fulfilling condition (3).

Note that condition (5) is strictly local and thus only valid if the perturbation is small enough such that higher-order terms $\mathcal{O}\left(|\mathrm{d} \varepsilon|^{3}\right)$ can be neglected. To keep this in mind, an equilibrium configuration will be called locally stable, if condition (5) is fulfilled. In most cases of material or structural stability analysis, it might indeed be physically reasonable to assume that the perturbations are small enough to neglect higherorder terms, even though this is normally not further validated. In the following, it will be seen that this is not necessarily the case when dealing with size effects of unstable metamaterials. For this, the minimal energy barrier separating two local minima is calculated explicitly, which gives a qualitative insight into how the structure behaves under finite perturbations. This problem has been solved for the von Neumann boundary condition in [23] and [24]. However, as shown in the following, the argumentation used therein can be easily transferred to the Dirichlet boundary value problem.

To calculate the minimal energy barrier, it is first necessary to further specify the potential $w$. For simplicity, the following non-convex piecewise quadratic function is used

$$
w(\varepsilon)= \begin{cases}\frac{1}{2} k_{1} \varepsilon^{2} & \varepsilon<\varepsilon_{b} \\ \frac{1}{2}\left(k_{1} \varepsilon_{b}\left(2 \varepsilon-\varepsilon_{b}\right)-k_{2}\left(\varepsilon-\varepsilon_{b}\right)^{2}\right) & \varepsilon_{b}<\varepsilon<\varepsilon_{h}, \\ \frac{1}{2}\left(k_{1} \varepsilon_{b}\left(2 \varepsilon-\varepsilon_{b}\right)-k_{2}\left(\varepsilon-\varepsilon_{h}\right)^{2}+k_{3}\left(\varepsilon_{h}-\varepsilon_{b}\right)\left(2 \varepsilon-\varepsilon_{h}-\varepsilon_{b}\right)\right) & \varepsilon_{h}<\varepsilon\end{cases}
$$

which corresponds to a trilinear stress strain behaviour (see Fig. 2) with limit strains $\varepsilon_{b}$ and $\varepsilon_{h}$, and stiffness $k_{1}>0, k_{2}<0$ and $k_{3}>0$

$$
\sigma(\varepsilon)=w^{\prime}(\varepsilon)= \begin{cases}k_{1} \varepsilon & \varepsilon<\varepsilon_{b} \\ k_{1} \varepsilon_{b}+k_{2}\left(\varepsilon-\varepsilon_{b}\right) & \varepsilon_{b}<\varepsilon<\varepsilon_{h} . \\ k_{1} \varepsilon_{b}+k_{2}\left(\varepsilon_{h}-\varepsilon_{b}\right)+k_{3}\left(\varepsilon-\varepsilon_{h}\right) & \varepsilon_{h}<\varepsilon\end{cases}
$$

With this potential, the local problem (2) has up to three different solutions for every unit cell which will be denoted by $\varepsilon_{I}, \varepsilon_{I I}$ and $\varepsilon_{I I I}$. Herein, $\varepsilon_{I}$ and $\varepsilon_{I I I}$ correspond to an unit cell in the positive stiffness range below 


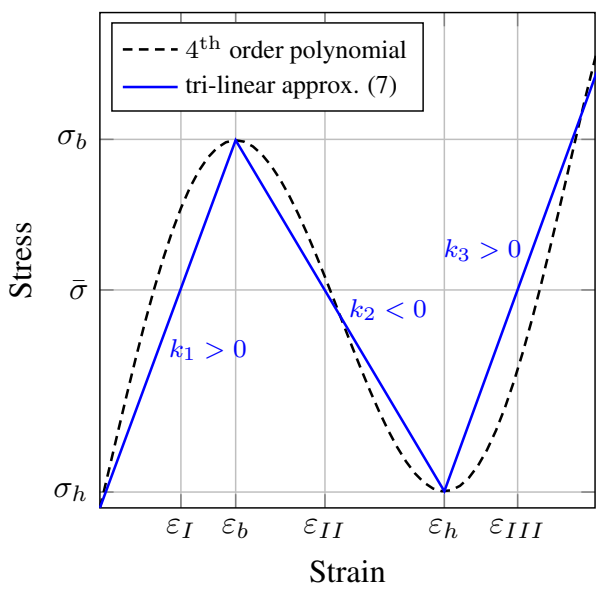

Fig. 2 Two different stress-strain relations corresponding to a non-convex potential: 4th-order polynomial (black dashed) used for the numerical investigation in Sect. 5 and the trilinear approximation (solid blue) used for the analytical investigation in Sect. 3

$\varepsilon_{b}$ and above $\varepsilon_{h}$ respectively, whereas solution $\varepsilon_{I I}$ corresponds to an unit cell in the negative stiffness range (see also Fig. 2). For a given external force $\bar{\sigma}$, these three solutions are calculated from (7)

$$
\begin{aligned}
\varepsilon_{I} & =\frac{\bar{\sigma}}{k_{1}}, \\
\varepsilon_{I I} & =\frac{\bar{\sigma}+\varepsilon_{b}\left(k_{2}-k_{1}\right)}{k_{2}}, \\
\varepsilon_{I I I} & =\frac{\bar{\sigma}+\varepsilon_{b}\left(k_{2}-k_{1}\right)+\varepsilon_{h}\left(k_{3}-k_{2}\right)}{k_{3}},
\end{aligned}
$$

where all three solutions only coexist in the non-trivial range $k_{1} \varepsilon_{b}+k_{2}\left(\varepsilon_{h}-\varepsilon_{b}\right) \leq \bar{\sigma} \leq k_{1} \varepsilon_{b}$. For stresses below this range $\left(\bar{\sigma} \leq k_{1} \varepsilon_{b}+k_{2}\left(\varepsilon_{h}-\varepsilon_{b}\right)\right), \varepsilon_{I}$ is the only solution, whereas for stresses above $\left(k_{1} \varepsilon_{b} \leq \bar{\sigma}\right)$, $\varepsilon_{I I I}$ is the only solution.

Within the non-trivial range, the solution space for the global problem is given by all possible combinations of unit cells having a strain $\varepsilon_{I}, \varepsilon_{I I}$ or $\varepsilon_{I I I}$. Since potential (6) is the same for all cells, the spatial order does not change the overall behaviour. Thus, a certain solution branch can be specified by three integers $p, q$ and $r$ (with $p+q+r=N$ ) giving the number of cells having a strain $\varepsilon_{I}, \varepsilon_{I I}$ and $\varepsilon_{I I I}$ respectively. For a specific configuration $\mathcal{C}:=(p, q, r)$, the overall behaviour is calculated from (3)

$$
\bar{\varepsilon}_{\mathcal{C}}(\bar{\sigma})=\frac{1}{N}\left(p \varepsilon_{I}+q \varepsilon_{I I}+r \varepsilon_{I I I}\right) .
$$

Together with (8)-(10), this can be inverted in order to get the overall stress-strain behaviour of a certain configuration $\mathcal{C}$

$$
\bar{\sigma}_{\mathcal{C}}(\bar{\varepsilon})=\frac{k_{1}\left(\varepsilon_{b}\left(k_{1}-k_{2}\right)\left(k_{3} q+k_{2} r\right)+k_{2}\left(\varepsilon_{h}\left(k_{2}-k_{3}\right) r+\bar{\varepsilon} k_{3} N\right)\right)}{p k_{2} k_{3}+q k_{1} k_{3}+r k_{1} k_{2}} .
$$

As proven in [23], there exists a critical number of unit cells $N_{\text {crit }}$ above which all equilibrium configurations with any unit cell in the negative stiffness range $(q \geq 1)$ are unstable, i.e. a saddle point or local maximum with $\partial_{i j} W$ negative definite. Clearly, the condition $N \geq N_{\text {crit }}$ is fulfilled for the analysis of size effects in the limit case $N \rightarrow \infty$. However, it will also be seen in the numerical results in Sect. 5 that $N_{\text {crit }}$ is typically a rather small value. Thus, it is sufficient for the following analysis to consider $\mathcal{C}=(i, 0, N-i)$ with $i=1, \ldots, N$ as the only stable equilibrium configurations for which $\bar{\sigma}^{\prime}(\bar{\varepsilon})>0$ holds.

With these preliminaries, it is now possible to show that the minimal energy barrier separating two local minima $\mathcal{C}_{1}=(i, 0, N-i)$ and $\mathcal{C}_{2}=(i-1,0, N-i+1)$ is obtained if one goes through the saddle point $\mathcal{C}_{s}^{1}=(i-1,1, N-i)$. The proof for this is based on the mountain pass theorem and can be performed in analogy to the von Neumann case given in [24]. For this the energy difference between $\mathcal{C}_{1}$ and all saddle points $\mathcal{C}_{s}^{t}=(i-t, t, N-i)$ with $t=1, \ldots, i$ is calculated. For the sake of demonstration, the following calculations are restricted to the case of $i=N$, whereas the result is qualitatively the same as for the general case, however 


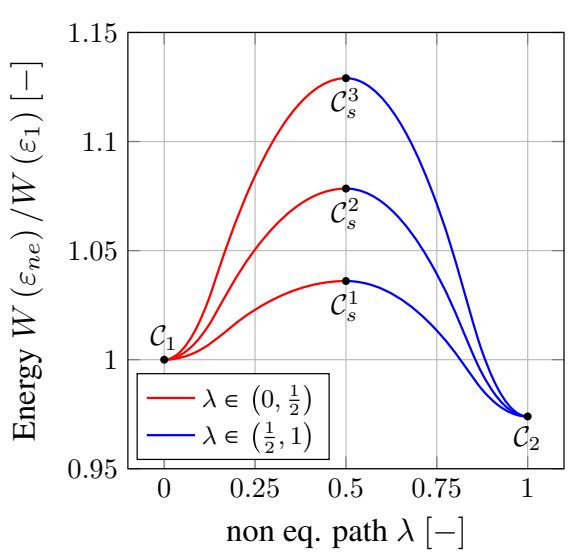

(a)

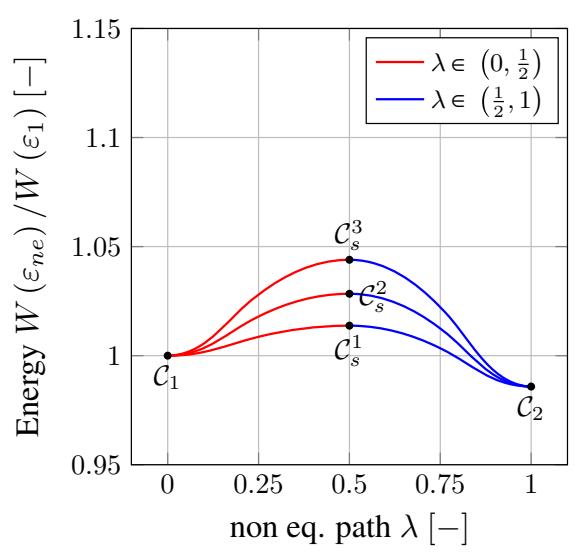

(b)

Fig. 3 Elastic energy for different non-equilibrium paths going from the stable equilibrium configuration $\mathcal{C}_{1}$ to the stable equilibrium configuration $\mathcal{C}_{2}$ for $N=40$ (a) and $N=100$ (b) cells for an external strain of $\bar{\varepsilon}=0.18$. The remaining parameters used for the evaluation are given in the "Appendix"

with much simpler expressions. Together with (8)-(10), the energy of a certain equilibrium configuration can be calculated from

$$
W(\boldsymbol{\varepsilon})=\frac{L}{N}\left(p W\left(\varepsilon_{I}\right)+q W\left(\varepsilon_{I I}\right)+r W\left(\varepsilon_{I I I}\right)\right)
$$

and thus, the energy barrier $\Delta W$ between $\mathcal{C}_{s}^{t}$ and $\mathcal{C}_{1}$ with $i=N$ can be calculated as

$$
\Delta W:=W\left(\mathcal{C}_{s}^{t}\right)-W\left(\mathcal{C}_{1}\right)=-\frac{\left(\bar{\varepsilon}-\varepsilon_{b}\right)^{2} k_{1}\left(k_{1}-k_{2}\right) L t}{2\left(k_{2}(N-t)+k_{1} t\right)} .
$$

From here, it follows that $\Delta W\left(t_{2}\right)<\Delta W\left(t_{1}\right)$ if $1 \leq t_{2}<t_{1} \leq N$, and thus, the smallest energy barrier is indeed given by $t=1$.

Equation (14) now permits the qualitative discussion of the perturbation resistance, without the need to neglect higher-order terms in the Taylor expansion (4). First of all, the energy barrier is reduced by increasing $\bar{\varepsilon}$ and vanishes for $\bar{\varepsilon}=\varepsilon_{b}$. A vanishing energy barrier corresponds to a loss of local stability according to (5). As will be seen by the numerical results in Sect. 5, this is true. Next, by keeping the overall structural size $L$ constant, the energy barrier is also reduced by increasing the number of unit cells $N(\Delta W \propto 1 / N)$ and thus also fully vanishes in the limit $N \rightarrow \infty$. This means that with an increasing number of cells, the energy barrier separating two local minima is getting smaller and smaller and the structure will become more sensitive to finite perturbations.

Finally, increasing the length $L$ with a fixed unit cell size $s$, also increases the total (external) energy. It is thus physically more relevant to look at the specific energy barrier as the ratio between the energy difference (14) and the overall energy $W\left(\mathcal{C}_{1}\right)$ :

$$
\Delta \bar{W}=\frac{\Delta W}{W\left(\mathcal{C}_{1}\right)}=-\frac{\left(\bar{\varepsilon}-\varepsilon_{b}\right)^{2}\left(k_{1}-k_{2}\right) t}{\bar{\varepsilon}^{2}\left(k_{2}(N-t)+k_{1} t\right)}
$$

from where the same size effects are found.

To further illustrate these results, one can calculate the elastic energy for the non-equilibrium path

$$
\boldsymbol{\varepsilon}_{\mathrm{ne}}= \begin{cases}\boldsymbol{\varepsilon}_{1}+\lambda\left(\boldsymbol{\varepsilon}_{s}^{t}-\boldsymbol{\varepsilon}_{1}\right), & \lambda \in\left(0, \frac{1}{2}\right) \\ \boldsymbol{\varepsilon}_{s}^{t}+\lambda\left(\boldsymbol{\varepsilon}_{2}-\boldsymbol{\varepsilon}_{s}^{t}\right), & \lambda \in\left(\frac{1}{2}, 1\right)\end{cases}
$$

going from $\mathcal{C}_{1}$ to $\mathcal{C}_{s}^{t}$ and then from $\mathcal{C}_{s}^{t}$ to $\mathcal{C}_{2}$, where $\varepsilon_{1}$ to $\varepsilon_{s}^{t}$ and $\varepsilon_{2}$ are the vectors with the corresponding strain components. This path is only in equilibrium for $\lambda=0, \lambda=\frac{1}{2}$ and $\lambda=1$ and is therefore only admissible if there exists an external perturbation according to (16). Furthermore, since (16) is linear it is fulfilling the boundary condition (3) and the elastic energy can be evaluated neglecting the second term in (1). 
In Fig. 3, the energy of this non-equilibrium path is plotted for $N=40$ and $N=100$ cells. As has been argued above, the energy barrier is given by the saddle configuration $\mathcal{C}_{s}^{t}$, whereas the path though $\mathcal{C}_{s}^{1}$ leads to the smallest possible energy barrier. Furthermore, it can be clearly seen how the energy barrier is reduced by increasing the number of cells.

The existence of a perturbation that exactly follows the minimum non-equilibrium path through the saddle configuration $\mathcal{C}_{s}^{1}$ is a strong assumption. However, since all other energy barriers are reduced as well, the probability of an earlier transformation is expected to increase by increasing the number of cells even if one applies a random perturbation.

To differentiate stability under finite perturbations from the local stability condition (5), an equilibrium configuration will be called globally (un)stable if the applied perturbation has an energy (larger) smaller than that given by Eq. (14). The exact perturbation mode is not considered, and it is assumed that the applied perturbation is indeed able to bring the structure through the saddle configuration $\mathcal{C}_{s}^{1}$ if it has a sufficient activation energy.

\section{Analytical investigation-the gradient enhanced continuum model}

The discrete model is compared with a corresponding gradient enhanced continuum model with a strain energy density

$$
w_{\nabla}\left(\varepsilon, \partial_{x} \varepsilon\right)=w(\varepsilon)+\frac{1}{2} A\left(\partial_{x} \varepsilon\right)^{2},
$$

where $w(\varepsilon)$ is the same as in (1), but with $\varepsilon_{i}$ substituted by the continuous strain $\varepsilon=\varepsilon(x)$. For the gradient part, the simplest possible extension, namely a quadratic uncoupled part with a gradient stiffness $A>0$, has been chosen. In here, the gradient part now plays the role of a localization limiter prescribing - through $A$-the length (area) where the material is in the softening phase with $\partial_{\varepsilon \varepsilon} w<0$. Thus, the gradient stiffness $A$ is somehow related to the unit cell size $s=L / N$ which prescribes the intrinsic softening length in the discrete model.

Stable equilibrium configurations of the gradient model are given by local minimizers of the overall potential

$$
W=\int_{0}^{L} w(\varepsilon)+\frac{1}{2} A\left(\partial_{x} \varepsilon\right)^{2}+\bar{\sigma}(\bar{\varepsilon}-\varepsilon) \mathrm{d} x+\bar{r}_{1} \partial_{x} \varepsilon(0)+\bar{r}_{2} \partial_{x} \varepsilon(L)
$$

In extension to the boundary condition with respect to the strain $\varepsilon$, higher-grade boundary conditions $\partial_{x} \varepsilon(0)=$ $\partial_{x} \varepsilon(L)=0$ with corresponding Lagrange multipliers $r_{1}$ and $r_{2}$ are now necessary. The latter boundary condition is chosen such that the gradient problem still contains the homogeneous (trivial) solution. This is consistent with the discrete problem where the solution is homogeneous until the first instability and subsequent localization. A further discussion of the physical relevance of the higher-grade boundary condition will be given later. The Euler-Lagrange equations follow from the first variation with respect to the displacement field $u$, and the Lagrange multipliers $\bar{\sigma}, \bar{r}_{1}$ and $\bar{r}_{2}$

$$
\begin{aligned}
& \partial_{x} \sigma_{\text {ref }}-A \partial_{x x x} \varepsilon=0, \\
& \bar{\sigma}=\sigma_{\text {ref }}(0)-A \partial_{x x} \varepsilon(0)=\sigma_{\text {ref }}(L)-A \partial_{x x} \varepsilon(L), \\
& \bar{r}_{1}=\bar{r}_{2}=A \partial_{x} \varepsilon(0)=A \partial_{x} \varepsilon(L)=0, \\
& \bar{\varepsilon}=\frac{1}{L} \int_{0}^{L} \varepsilon \mathrm{d} x,
\end{aligned}
$$

where the referential stress $\sigma_{\text {ref }}:=\partial_{\varepsilon} w(\varepsilon)$ has been introduced.

\subsection{Stability analysis}

Analogous to the stability of the discrete model, an equilibrium configuration of the continuum model is locally stable if the second variation is positive. Together with the boundary conditions, the stability condition is retrieved from (18) as

$$
\delta_{u u} W=\int_{0}^{L} \partial_{\varepsilon \varepsilon} w(\varepsilon)\left(\delta_{u} \varepsilon\right)^{2}+A\left(\partial_{x} \delta_{u} \varepsilon\right)^{2} \mathrm{~d} x>0
$$


This condition can be seen as a special case of the stability problem investigated in [26] and thus can be further evaluated by following the method presented therein. For this, the variation field is written as a Fourier series

$$
\delta u=\sum_{n=1}^{\infty} \delta u_{n} \sin \left(\frac{n \pi x}{L}\right)
$$

Note that this ansatz is compatible with the essential boundary conditions in (20), and thus, no further conditions are imposed on the Fourier coefficients $\delta u_{n}$.

Together with the orthogonality relation

$$
\int_{0}^{L} \sin \left(\frac{n \pi x}{L}\right) \sin \left(\frac{k \pi x}{L}\right) \mathrm{d} x=\frac{L}{2} \delta_{n k}
$$

the second variation from (21) can be written as

$$
\delta_{u u} W=\sum_{n=1}^{\infty} \sum_{k=1}^{\infty} \int_{0}^{L} \partial_{\varepsilon \varepsilon} w(\varepsilon) \cos \left(\frac{n \pi x}{L}\right) \cos \left(\frac{k \pi x}{L}\right) \frac{n k \pi^{2}}{L^{2}} \mathrm{~d} x \delta u_{n} \delta u_{k}+A \frac{L}{2} \sum_{n=1}^{\infty}\left(\frac{n \pi}{L}\right)^{4} \delta u_{n}^{2} .
$$

For the homogeneous solution $\left(\partial_{\varepsilon \varepsilon} w(\varepsilon)=\right.$ const .), this can, again using the orthogonality relation, be further simplified to

$$
\delta_{u u} W=\sum_{n=1}^{\infty} \underbrace{\left(\partial_{\varepsilon} \sigma_{\text {ref }}+A\left(\frac{n \pi}{L}\right)^{2}\right)}_{=: \eta_{n}} \frac{n^{2} \pi^{2}}{2 L} \delta u_{n}^{2}>0 .
$$

Since the Fourier coefficients are linearly independent, the homogeneous solution is locally stable if the coefficients $\eta_{n}$ are positive for all $n \geq 1$. However, $A$ is positive by definition, and thus, $\eta_{1} \geq 0$ is necessary and sufficient for local stability. From here, it can be concluded that a homogeneous solution with a strain corresponding to state $I$ or $I I I$ (see Fig. 2) is unconditionally stable, since $\partial_{\varepsilon} \sigma_{\text {ref }} \geq 0$. A homogeneous solution with a strain corresponding to state $I I$ (negative stiffness) is locally stable, if

$$
-A\left(\frac{\pi}{L}\right)^{2} \leq \partial_{\varepsilon} \sigma_{\text {ref }} \leq 0
$$

This condition permits the discussion of stability size effects as predicted by the gradient model. For this, one compares two structures with lengths $L_{2}>L_{1}$. From (26), it follows that the homogeneous solution

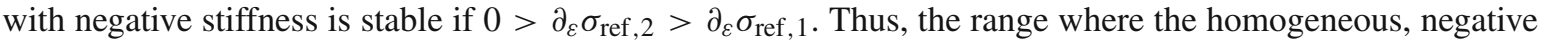
stiffness solution is stable decreases with the length of the structure. In the limit $L \rightarrow \infty$, one has $\partial_{\varepsilon} \sigma_{\text {ref }} \rightarrow 0$ as stability condition: The homogeneous negative stiffness solution becomes completely unstable. By rearranging condition (26), one gets the maximum structural length $L_{\max }$ until which the homogeneous solution is stable

$$
L_{\max }=\pi \sqrt{\frac{A}{-\min \partial_{\varepsilon} \sigma_{\mathrm{ref}}}},
$$

where $\min \partial_{\varepsilon} \sigma_{\text {ref }}$ is the minimal stiffness within the negative stiffness range. Furthermore, the length $L_{\max }$ from (27) is also equivalent to a minimal length for which the structure will loose stability and thus show a bifurcation into an inhomogeneous solution.

Finally, note that the stability analysis in this section has been done without reference to the exact form of the reference potential $w(\varepsilon)$, and thus conditions (25)-(27) are valid for arbitrary non-convex reference potentials. 


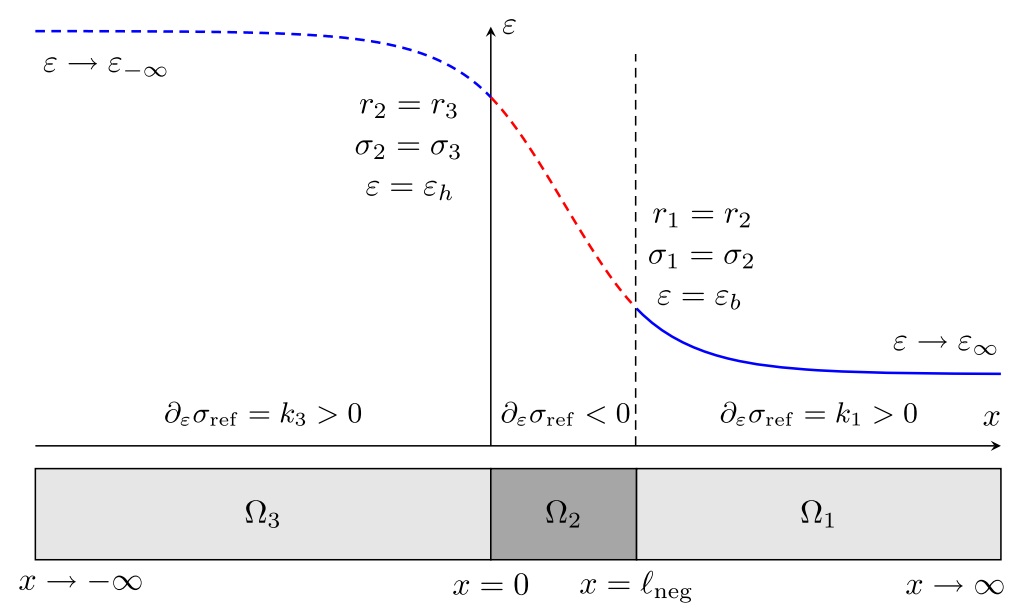

Fig. 4 Illustration of the strain distribution of the infinite problem together with the softening zone of size $\ell_{\text {neg }}$ with a negative stiffness $\partial_{\varepsilon} \sigma_{\text {ref }}<0$, its discretization according to (6) and the coupling conditions given in (29)-(30)

\subsection{Analytical estimate for the gradient stiffness}

Going from the discrete model to the gradient enhanced continuum model, the reference potential $w(\varepsilon)$ is retained. However, since the discrete model does not contain any non-local interactions, the gradient stiffness $A$ is not defined up to now. As stated before, the gradient stiffness needs to be related to the unit cell size in the discrete model, prescribing the length of softening material. Thus, the gradient stiffness $A$ will be defined here by the condition that the gradient model shows the same ratio $L / \ell_{\text {neg }}$ between overall structure size $L$ and softening material length $\ell_{\text {neg }}$ with $\partial_{\varepsilon} \sigma_{\text {ref }}(x)<0$ as the discrete model (see Fig. 4). In the discrete model, this ratio is simply given by the number of cells $N=L / s$.

Defining the gradient stiffness like this requires the relation between the softening length $\ell_{\text {neg }}$ and gradient stiffness $A$. To obtain this, a solution to the boundary value problem (19)-(20) is approximated adopting the approach from [11]. For this, two approximations are introduced; first the reference potential is again approximated as in (6), and second, instead of solving the boundary value problem for a finite length, the differential equation (19) is solved for the infinite problem. Furthermore, without loss of generality, the solution can be discretized into three regions $\Omega_{1}, \Omega_{2}$ and $\Omega_{3}$ (Fig. 4), where $\Omega_{1}\left(\Omega_{3}\right)$ is the region with a strain $\varepsilon<\varepsilon_{b}$ $\left(\varepsilon>\varepsilon_{h}\right)$ and $\Omega_{2}$ is the region of length $\ell_{\text {neg }}$ having a strain within the negative stiffness region $\left(\varepsilon_{h}>\varepsilon>\varepsilon_{b}\right)$. In general, other phase distributions with more than three phases would be possible; however as shown in [26], these are unstable and thus not considered here. By this discretization, the nonlinear differential equation (19) can be split into the following coupled system of linear differential equations

$$
k_{i} \partial_{x} \varepsilon_{i}-A \partial_{x x x} \varepsilon_{i}=0 \quad \forall x \in \Omega_{i}, \quad i=1,2,3 \text { (no sum). }
$$

Equations (19) and therefore also (28) are derived from (18) by use of partial integration and thus only valid for a continuous strain $\varepsilon$. This requirement serves as a first boundary (or interface) condition for the system (28)

$$
\varepsilon_{1}(0)=\varepsilon_{2}(0)=\varepsilon_{h}, \quad \varepsilon_{2}\left(\ell_{\text {neg }}\right)=\varepsilon_{3}\left(\ell_{\text {neg }}\right)=\varepsilon_{b} .
$$

Further coupling conditions are obtained from the continuity of stress and higher stress resulting from a split of (18) into the three regions $\Omega_{i}$. The higher stress is given by $r=A \partial_{x} \varepsilon$, and thus, the related interface condition is expressed as

$$
\partial_{x} \varepsilon_{1}(0)=\partial_{x} \varepsilon_{2}(0), \quad \partial_{x} \varepsilon_{2}\left(\ell_{\text {neg }}\right)=\partial_{x} \varepsilon_{3}\left(\ell_{\text {neg }}\right) .
$$

Together with (29), the continuity of stress $\sigma=\partial_{\varepsilon} w(\varepsilon)-A \partial_{x x} \varepsilon$ leads to the third interface condition given by

$$
\partial_{x x} \varepsilon_{1}(0)=\partial_{x x} \varepsilon_{2}(0), \quad \partial_{x x} \varepsilon_{2}\left(\ell_{\text {neg }}\right)=\partial_{x x} \varepsilon_{3}\left(\ell_{\text {neg }}\right) .
$$


Additionally, it is required that the strain remains finite in the limits

$$
\lim _{x \rightarrow \infty} \varepsilon_{1}(x)=\varepsilon_{\infty} \text { and } \lim _{x \rightarrow-\infty} \varepsilon_{1}(x)=\varepsilon_{-\infty} .
$$

Since $k_{1}>0, k_{3}>0$ and $k_{2}<0$, the general solution for (28) is

$$
\begin{aligned}
& \varepsilon_{i}(x)=C_{1}^{i} \exp \left(\omega_{i} x\right)+C_{2}^{i} \exp \left(-\omega_{i} x\right)+C_{3}^{i} \quad \forall x \in \Omega_{i}, \quad i=1,3 \\
& \varepsilon_{2}(x)=C_{1}^{2} \sin \left(\omega_{2} x\right)+C_{2}^{2} \sin \left(\omega_{2} x\right)+C_{3}^{2} \quad \forall x \in \Omega_{2},
\end{aligned}
$$

whereas $\omega_{i}:=\sqrt{\left|k_{i}\right| / A}$ has been defined.

Equations (29)-(34) can be seen as an eigenvalue problem for the eigenvalue $\ell_{\text {neg. }}$. After eliminating all coefficients $C_{i}^{j}$ by conditions (29)-(32), the following condition remains to be fulfilled

$$
\left(\varepsilon_{h}-\varepsilon_{-}\right)\left(\sin \left(\omega_{2} \ell_{\text {neg }}\right)\left(\omega_{3} \omega_{1}-\omega_{2}^{2}\right)+\cos \left(\omega_{2} \ell_{\text {neg }}\right)\left(\omega_{2} \omega_{1}+\omega_{3} \omega_{2}\right)\right)=0 .
$$

This can be fulfilled if either the term in the second bracket vanishes, or if $\varepsilon_{h}=\varepsilon_{-\infty}$. The latter condition leads to the homogeneous solution $\varepsilon \equiv \varepsilon_{h}$, and thus, the eigenvalues for an inhomogeneous solution are given by

$$
\ell_{\text {neg }}=\frac{1}{\omega_{2}}\left(\arctan \left(\frac{\omega_{2}\left(\omega_{3}+\omega_{1}\right)}{\omega_{2}^{2}-\omega_{3} \omega_{1}}\right)+n \pi\right) \text { with } n \in \mathbb{N} .
$$

For the physically relevant solution, $n$ has to be chosen according to $\omega_{1}, \omega_{2}$ and $\omega_{3}$ such that $\ell_{\text {neg }}$ is positive and as small as possible, since this is related to a softening length with the smallest possible strain energy and thus expected to be the stable configuration. Considering this, the required relation between gradient stiffness $A$ and softening size $\ell_{\text {neg }}$ can be obtained by solving (36) for $A$

$$
A=\left(\frac{\sqrt{\left|k_{2}\right|} \ell_{\mathrm{neg}}}{\arctan \left(\frac{\sqrt{\left|k_{2}\right|}\left(\sqrt{k_{3}}+\sqrt{k_{1}}\right)}{\left|k_{2}\right|-\sqrt{k_{3}} \sqrt{k_{1}}}\right)+n \pi}\right)^{2}
$$

where $n=0$ if $\omega_{2}^{2}>\omega_{3} \omega_{1}$ and $n=1$ if $\omega_{2}^{2}<\omega_{3} \omega_{1}$. In case of equality $\left(\omega_{2}^{2}=\omega_{3} \omega_{1}\right)$, the gradient stiffness is directly obtained from (35) as

$$
A=\left(\frac{2 \sqrt{\left|k_{2}\right|} \ell_{\text {neg }}}{\pi}\right)^{2} .
$$

\section{Numerical results}

In this section, the analytical results from Sects. 3 and 4 are complemented with numerical results from both models. For this, the potential $w(\varepsilon)$ in (1) and (17) is chosen to be a 5th-order polynomial and the gradient stiffness $A$ is calculated from (36) such that the ratio between softening zone and overall structure size is finite and corresponds to a discrete model with $N=L / s=L / \ell_{\text {neg }}$ cells, where $s$ is the cell size. The exact form of the potential $w(\varepsilon)$ as well as all other parameters is summarized in the "Appendix."

For the discrete problem, equilibrium configurations are found by applying the Newton-Raphson method together with an arc-length constraint as has been detailed in [17]. Within this solution scheme, the local stability is investigated by numerically calculating the eigenvalues of $\partial_{i j} W$. The gradient boundary value problem in turn is solved by the shooting method. Herein, finding the homogeneous solution is trivial. The localized solution can be found using the analytical approximation from Sect. 4.2 as a starting value. Local stability of the homogeneous solution is evaluated by the analytical condition (26), and for the localized solution, a numerical approximation of (24) is used.

Additionally, the gradient problem is investigated together with a global stability analysis in Sect. 5.2. The global stability analysis is motivated by [24] as well as the related analytical investigations in Sect. 3 and is done as follows: For every equilibrium configuration, it is first checked if there exists a second locally 


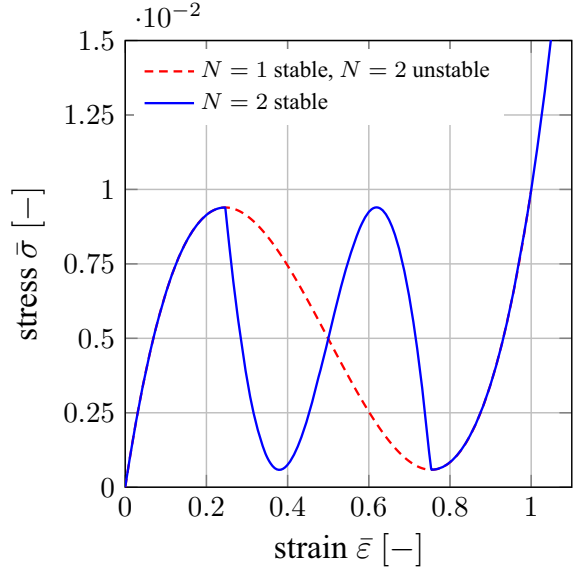

(a)

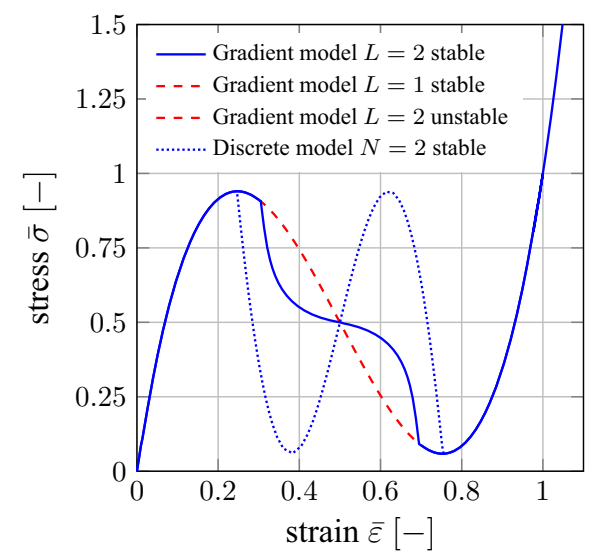

(b)

Fig. 5 Comparison of numerical results from the discrete model a for $N=1$ and $N=2$ cells with a corresponding gradient enhanced model $\mathbf{b}$ with a length of $L=1$ and $L=2$. The two red dashed lines in (a) and (b) are superimposed

stable equilibrium configuration that has a smaller strain energy and fulfils the boundary condition. If yes, the minimal energy barrier between these two is calculated from the saddle configuration with $t=1$ (see Sect. 3). If this energy barrier is smaller than a given perturbation energy $W_{\text {pert }}$ the equilibrium configuration is globally unstable and the structure changes to the locally stable equilibrium configuration with the smaller strain energy. If there are more than two locally stable equilibrium configurations that fulfil the boundary conditions the above procedure is repeated until a globally stable equilibrium configuration is found.

\subsection{Local stability criterion}

In what follows, the discrete and the gradient enhanced models are compared for an increasing structural size $L$, keeping both the unit cell size $s=L / N$ and the gradient stiffness $A$ constant.

Figure 5a shows the behaviour of the discrete model for $N=1$ and $N=2$ unit cells. As expected, solving the discrete problem for only one cell exactly reproduces the stress-strain behaviour derived from the local potential $w(\varepsilon)$. This is different for two and more cells: Stability requires that only one cell has a state with a negative stiffness; thus, the homogeneous solution becomes unstable and the two cells are passing the negative stiffness range one after the other. However, this transformation process still results in a smooth (i.e. continuous) effective behaviour for $N \leq 2$. Comparing this with the gradient model in Fig. $5 \mathrm{~b}$, the exact same behaviour is observed for $L=1$. The homogeneous solution is completely stable and there is no bifurcation observed. However, in contrast to the discrete model, this is not an obvious fact, since in a classical model (without the gradient term) a bifurcation following the Maxwell rule [29,33] would be expected. Consequently, the stabilization of the homogeneous solution is a consequence of the gradient part which restricts the size of the softening zone. If the structure is smaller than a critical $L_{\mathrm{Max}}$ the energy required for a smaller softening zone is simply too high compared to the homogeneous solution (see also Eq. (27)).

As expected from the stability analysis in Sect. 4.1, this behaviour changes with increasing length $L$. For $L=2$, there is a certain range where the homogeneous solution becomes unstable and bifurcates into an inhomogeneous solution. This agrees well with the prediction from Eq. (27) which in this case gives a critical length of $L_{\max }=1.4$. As in the discrete model, the reaction force for the inhomogeneous solution deviates from the referential stress (see also Eq. 20). However, in the gradient model the reaction force does not go through the same sequence of negative and positive stiffness branches related to the softening within the individual unit cells in the discrete set-up but instead shows a smooth monotonic behaviour. This is related to the fact that the continuum model does not obey the same discrete phase transitions as in the discrete model, but rather shows a smooth propagation of the softening region from one end to the other (Illustrated for the case of $L=3$ in Fig. 6c).

Comparing the stable equilibrium path from the discrete model for $N=2$ with the gradient model for $L=2$ in Fig. 5 b, it can be seen that bifurcation into the inhomogeneous solution is delayed in the gradient model. This delay can be attributed to the continuous strain profile of the gradient model compared to the 


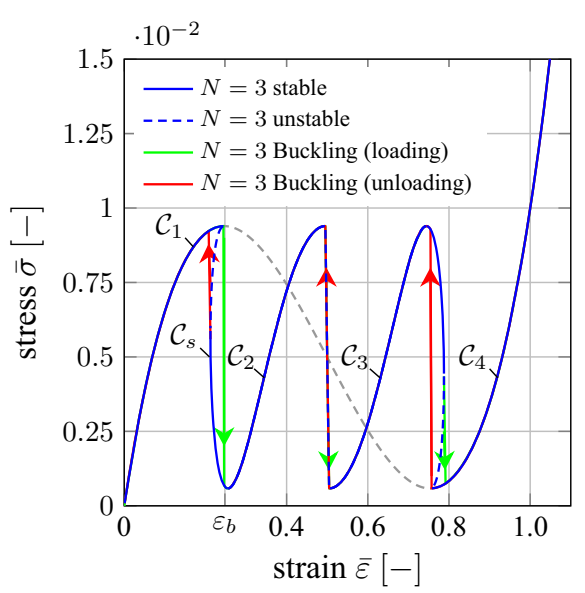

(a)

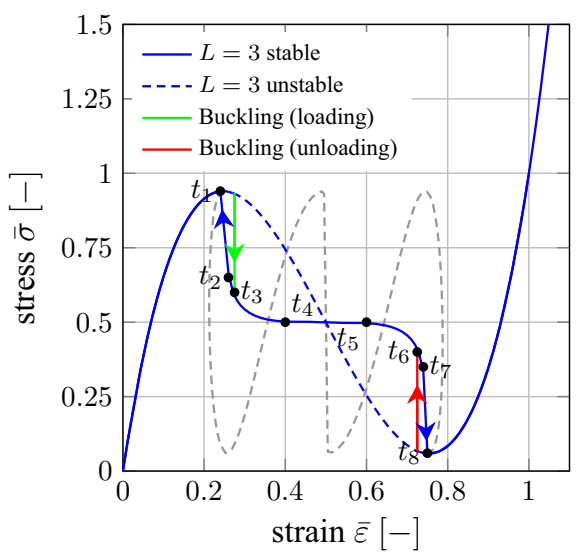

(b)

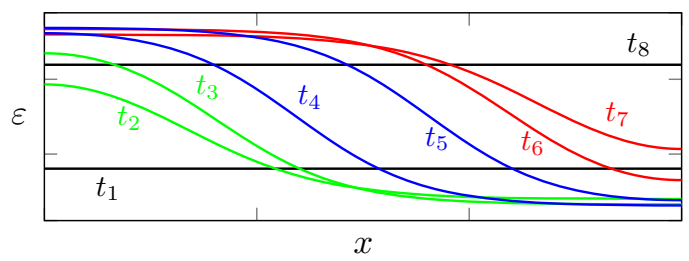

(c)

Fig. 6 Comparison of numerical results from the discrete model a for $N=3$ with a corresponding gradient enhanced model $\mathbf{b}$ with a length of $L=3$. Stable (unstable) equilibrium configurations are given by solid (dashed) blue lines, abrupt buckling under loading (unloading) is indicated by green (red) arrows. Selected strain profiles from the gradient model are illustrated in (c) for the different timesteps $t_{1}$ to $t_{8}$ as marked in (b)

discrete strain distribution of the discrete model. As can be seen from Fig. 4 and also Fig. 6c, the strain outside of the softening zone $\Omega_{2}$ varies continuously towards the boundaries where $\partial_{x} \varepsilon=0$ is prescribed. This deviation from the homogeneous solution requires some additional energy which delays the localization. However, this argumentation also anticipates that the influence of this transition zone from the softening to the quasi-homogeneous strain vanishes by increasing the size $L$. As will be seen in the following (compare Figs. $6 \mathrm{~b}, 7 \mathrm{~b}, 8 \mathrm{~b})$, this is the case and the delay in bifurcation is not seen any more for $L=40$ in Fig. $8 \mathrm{~b}$. This behaviour can also be rationalized by Eq. (26) which exactly predicted that the homogeneous solution with a negative stiffness is gradually loosing stability and in the limit the complete negative stiffness branch is unstable.

With $N=3$ unit cells, the bifurcation behaviour changes fundamentally. As shown in Fig. 6a, there are now several stable equilibrium configurations for the same external strain and the configuration $\mathcal{C}_{s}=(2,1,0)$ with one cell in the negative stiffness region becomes partially unstable. Beyond the stress maximum at $\varepsilon=\varepsilon_{b}$ (see Fig. 6a), the energy barrier separating $\mathcal{C}_{1}=(3,0,0)$ and $C_{2}=(2,0,1)$ vanishes as has been argued in Sect. 3. Thus, passing the limit point at $\varepsilon_{b}$, one of the cells buckles suddenly changing from $\mathcal{C}_{1}$ to $\mathcal{C}_{2}$.

For $\varepsilon=\varepsilon_{b}, \mathcal{C}_{1}$ has less energy than $\mathcal{C}_{2}$, thus changing from $\mathcal{C}_{1}$ to $\mathcal{C}_{2}$, a portion of the strain energy is released. This energy is first transformed into kinetic energy of local vibrations which are subsequently damped by for example the viscosity of the constituent material. Due to this, a loading-/unloading cycle passing $\varepsilon_{b}$ leads to an overall hysteresis. How the dissipation process takes place in detail has been discussed in the literature $[17,34]$ and will not be further detailed in the current investigation. Analogous processes take place under further loading during the transformation from $\mathcal{C}_{2}=(2,0,1)$ to $\mathcal{C}_{3}=(1,0,2)$ and $\mathcal{C}_{3}$ to $\mathcal{C}_{4}=(0,0,3)$ and under unloading similar transformations take place in the inverse order.

The behaviour of the corresponding gradient model (Fig. 6b) is similar in some respect. Passing $\varepsilon_{b}$ a point is reached where the initiation of the inhomogeneous solution occurs suddenly and an overall dissipative behaviour is observed. However, in contrast to the discrete model where there is an abrupt transformation corresponding to the buckling of every individual cell as $\varepsilon$ is increased, there are only two such abrupt transformations in the gradient model. These two abrupt transformations now correspond to the sudden initiation of the 


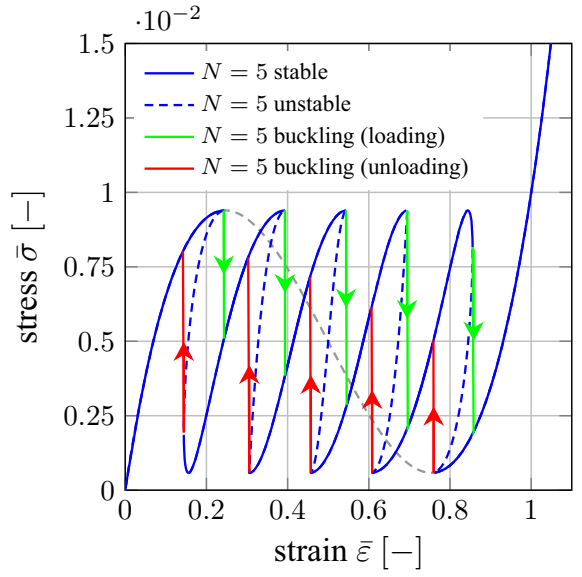

(a)

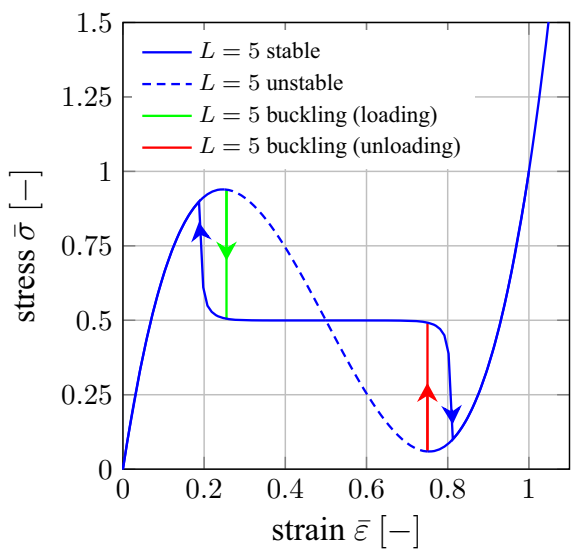

(b)

Fig. 7 Comparison of numerical results from the discrete model a for $N=5$ with a corresponding gradient enhanced model $\mathbf{b}$ with a length of $L=5$. Stable (unstable) equilibrium configurations are given by solid (dashed) blue lines; abrupt buckling under loading (unloading) is indicated by green (red) arrows

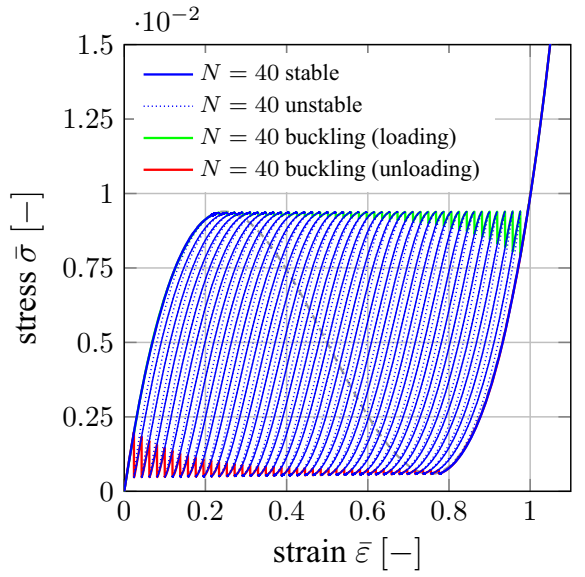

(a)

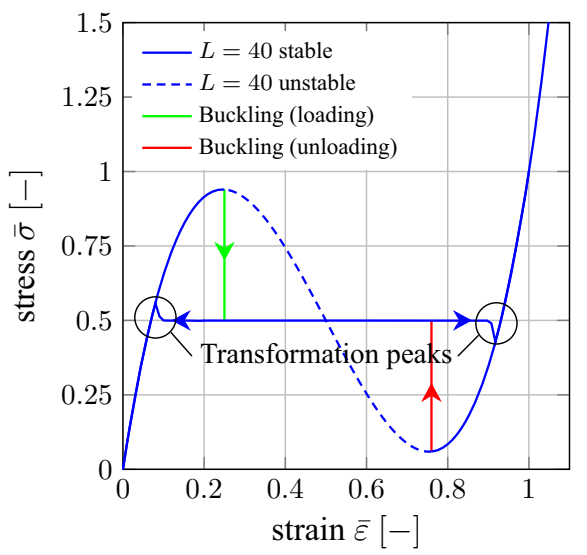

(b)

Fig. 8 Comparison of numerical results from the discrete model a for $N=40$ with a corresponding gradient enhanced model b with a length of $L=40$. Stable (unstable) equilibrium configurations are given by solid (dashed) blue lines; abrupt buckling under loading (unloading) is indicated by green (red) lines

inhomogeneous solution and the sudden transformation of the inhomogeneous solution back to a homogeneous solution with $\varepsilon>\varepsilon_{h}$ when the softening region reaches the other end (see Fig. 6c). The origin of the energy dissipation occurring during this transformation is the same as in the discrete model: At the transformation strain, the inhomogeneous solution has less energy than the homogeneous solution; thus changing from one to the other, a portion of the strain energy is released. An earlier transformation is not possible since the homogeneous and the inhomogeneous solutions are separated by a finite energy barrier. In between the two abrupt transformations, the softening zone continuously propagates through the structure, and thus, the reaction force shows a smooth behaviour without any corresponding energy release.

By further increasing the number of cells or the length of the structure (see Figs. 7, 8), the observed behaviour does not change qualitatively and only becomes more pronounced. As expected from the discussion of (26), the negative stiffness region becomes completely unstable in both models.

\subsection{Global stability criterion}

Adding a finite perturbation energy in a global stability analysis includes a second size effect which overlays with the size effects observed in the previous section. As predicted in Sect. 3 (Eq. (14)), the required energy 


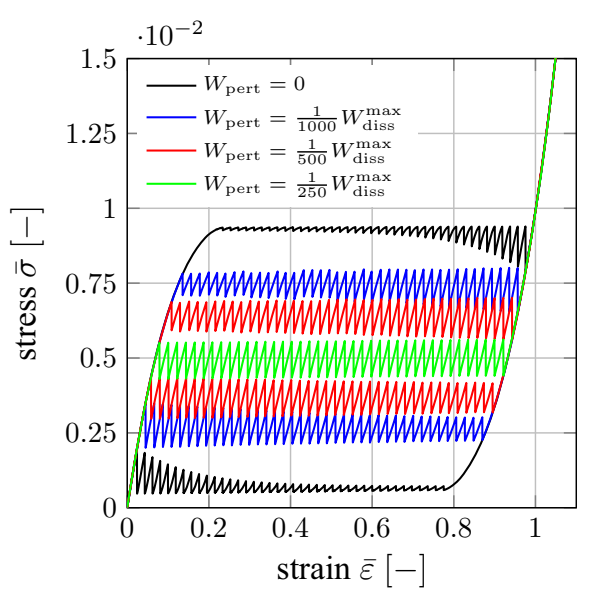

(a)

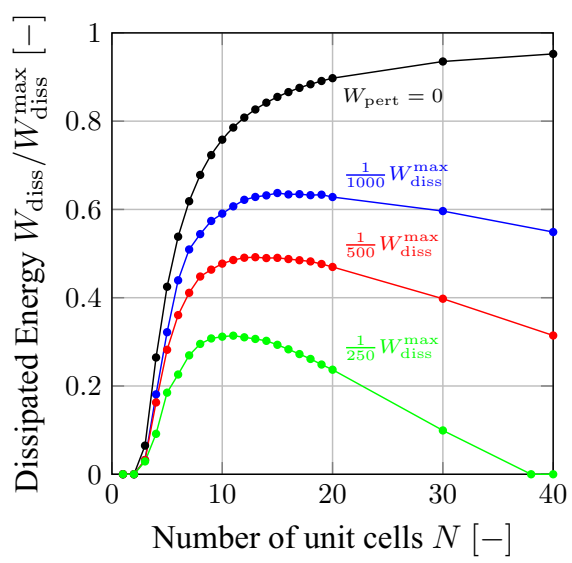

(b)

Fig. 9 Size effects predicted from a numerical solution of the discrete model together with a global stability criterion. The influence of a finite perturbation energy $W_{\text {pert }}$ on the loading/unloading path for $N=40$ cells (a) and the influence of the number of cells $N$ on the predicted energy dissipation for different perturbation energies $W_{\text {pert }}$ (b)

barrier for an earlier transformation is reduced by increasing the strain towards $\varepsilon_{b}$, the strain where the stress strain behaviour of the unit cell starts to decrease. Conversely, this means that the strain at which an earlier transformation is possible is reduced by increasing the perturbation energy.

This behaviour is exemplarily illustrated for $N=40$ cells in Fig. 9 where the loading and unloading path is plotted for different perturbation energies $W_{\text {pert }}$. In here, the perturbation energy has been normalized with respect to $W_{\text {diss }}^{\max }$ which is the maximum possible energy dissipation which would be obtained from a local stability condition in the limit of $N \rightarrow \infty$ or by a force controlled loading/unloading cycle. Clearly, the hysteresis and thus also the energy dissipation are reduced by increasing the perturbation energy. For a value of $W_{\text {pert }}=W_{\text {diss }}^{\max } / 250$, loading and unloading follows paths become indistinguishable and the energy dissipation appears to vanishes completely.

How the energy dissipation evolves with the number of cells is summarized in Fig. 9b. Without considering a finite perturbation energy, the energy dissipation monotonically increases towards the maximum possible value. Adding a finite perturbation energy, this size effect is overlaid with a second size effect which effectively leads to a non-monotonically energy dissipation. Once reaching zero energy dissipation further increasing the number of cells obviously does not change the hysteresis size any more. This behaviour is also consistent with the analytical predictions from Sect. 3.

\section{Discussion}

First mentioned in 1875, the Maxwell rule [29] is often used to describe phase transformation in systems with a non-convex potential. The Maxwell rule states that the negative stiffness region is inadmissible and thus passing it is only possible by a phase separation. The effective stress-strain curve bifurcates into the so-called Maxwell line which separates the local stress-strain curve into two equal areas. The validity of the Maxwell rule has been intensively discussed for liquid-gaseous phase transformations (see for example [35] and the references therein). For solids, a phase transformation following the Maxwell line can be obtained as a local minimizer of the strain energy potential of a classical continuum, as has been mathematically validated in [33]. However, the instability of the negative stiffness branch clearly depends on the boundary conditions and interestingly the negative stiffness branch can be observed experimentally if the kinematics are properly constrained. This has been shown for low carbon steel and NiTi laminated tensile samples in [36] which showed a nonmonotonic behaviour following the negative stiffness branch, whereas the unconstrained samples showed a transformation on the Maxwell line.

Here, the discrete model predicts a hysteresis and no transformation on a Maxwell line. This suggests that the Maxwell rule does not fully apply for unstable microstructures with a moderate ratio between overall structural size and unit cell size. This is further confirmed by experimental results that also show a hysteresis and thus a behaviour consistent with the predictions of the discrete model [16]. In the infinite limit, the gradient 
model in turn partially reproduces the prediction of the Maxwell rule, but it is not able to fully reproduce the behaviour of the discrete model. This leads to the question of the applicability and similarities of both models.

Noting first that the discrete model and the gradient continuum model are qualitatively and quantitatively consistent with respect to stability size effects in the sense that in both cases the homogeneous negative stiffness solution gradually loses stability with an increased structural size. Loss of stability is related to the bifurcation into an inhomogeneous solution initiated by a localization and subsequent propagation of the softening region. In both models, the hysteresis is related to an energy release occurring during an abrupt transformation from a higher to a lower energy level. However, even though the mechanisms leading to the hysteresis are comparable and the corresponding size effect is qualitatively the same, the hysteresis size is completely different. As indicated previously, the reason for this is that the gradient model only shows an abrupt transformation with a corresponding energy release during the initiation of a localization and not during the (smooth) propagation of the softening region.

Comparing the two models based on their model definition (1) and (18), one expects that the two models are the same in the limit of $N, L \rightarrow \infty$. In (1), one can substitute the sum operator with an integral and in (18) the gradient contribution does not change with the length $L$; thus, one can neglect its contribution in the infinite limit. The reason that the numerical results presented here do not approach each other for a finite size is twofold:

First, according to Eq. (14) or (15) the specific energy barrier preventing an earlier transformation on a Maxwell-like line scales with $1 / N$ and only vanishes in the limit $N \rightarrow \infty$. The same holds for the stability; as long as the energy barrier is finite, the structure is said to be locally stable according to condition (5). On the contrary, if one includes a finite activation energy assuming that there are external fluctuations which can help to overcome a finite energy barrier, global stability is lost, and an earlier transformation on a Maxwell-like line becomes possible (see Sect. 5.2, and also [23] and [24] for further illustration). In particular this behaviour is qualitatively independent of the portion of the activation energy, there will always be a number of unit cells, i.e. a structural size, according to (15) for which an earlier transformation is possible for a certain external strain $\bar{\varepsilon}$. The same argumentation can be applied to the gradient model, where an earlier initiation of the localization is expected if additional activation energy is included. Thus, the two models can only approach each other for finite sizes if one includes finite fluctuations.

Second, even including additional activation energy, the gradient model still shows an inconsistency to both the Maxwell line and the discrete model. This inconsistency is observed in a small transformation peak (marked in Fig. 8b) at the bifurcation point. As seen from Figs. 5, 6, 7 and 8, the size of this transformation peak does indeed get smaller with an increased length. However, as has been proven in [25], it does not vanish in the limit $L / \ell_{\text {neg }} \rightarrow \infty$.

This leads to the question if there is a physical mechanism that can be related to the transformation peak? In the literature, the transformation peak is typically related to two different mechanisms. First, it has been related to the different forces needed for the initiation and continuation of dislocation motion in [37] and [38]. This difference is related to the diffusion of atoms in the neighbourhood of the dislocations and leads to the formation of so-called Lüders-bands [39,40] and [11]. A second explanation is possible by analysing a discrete non-local model. In contrast to the discrete local model investigated in this paper, a discrete non-local model shows the same transformation peak due to the non-local interactions [23] and [25]. Thus, it is also possible to relate the transformation peak to the non-local interactions which for example occur at the atomistic scale.

Obviously, the first argumentation does not apply to the unstable microstructures investigated here. At first glance, the second argumentation does not make any sense in the case of the investigated one-dimensional discrete model either, since this model is strictly local and there is no physical reason to include non-local interactions. However, this is different if one investigates in real two- or three-dimensional structures where a delayed bifurcation can be observed due to a constraining effect of the boundary condition (see $[5,41]$ ). The same holds for the gradient model where the boundary condition on the gradient $\partial_{x} \varepsilon$ influences how a localization is initiated at the boundary. As seen in Fig. $6 \mathrm{c}$, choosing $\partial_{x} \varepsilon=0$ at the boundaries forces the localization profile to remain flat at the boundaries. This prevents the localization from initiating gradually and thus is the reason why a small transformation peak remains. This mechanism is analogue to the delay in bifurcation that has been observed and discussed for $L=2$ in Sect. 5.1 Similar boundary effects are known from the characterization of foams and other microstructured materials $[6,15,28,42,43]$ where higher-order boundary conditions are used to model stiffening size effects. 


\section{Summary}

Unstable microstructures in metamaterials show some interesting effective properties making them a promising alternative to well-established materials used for example as crash absorbers [16].

This paper presented a first step towards a continuum model of these unstable metamaterials by investigating a one-dimensional gradient enhanced continuum model. This model is capable of modelling size effects with respect to stability; however, it cannot quantitatively capture size effects with respect to energy dissipation since these are related to the discrete phase transitions.

Furthermore, this paper has demonstrated that a classical stability analysis might not be sufficient and a finite perturbation analysis becomes necessary to evaluate global stability of unstable metamaterials. Especially, this seems to be necessary to get a smooth transition of the predicted size effects going from the discrete to the gradient enhanced continuum model.

Acknowledgements This project has been financially supported by the Hector Fellow Academy through the project "Mechanical Metamaterials" and by the Deutsche Forschungsgemeinschaft (DFG, German Research Foundation) under Germany's Excellence Strategy via the Excellence Cluster 3D Matter Made to Order (EXC-2082-390761711). This support is gratefully acknowledged. P.G. also thanks the Fraunhofer Cluster of Excellence Programmable Materials for support.

\section{Appendix: Parameters}

The calculations in this paper have been done without reference to any experimental results, and thus, the parameters used for the simulation are only fictitious values chosen to get qualitative insight. However, to make sure the results are reproducible the parameters are summarized in the following. For the numerical simulations in Sect. 5, the stress-strain behaviour is chosen to be of 5th-order

$$
\sigma(\varepsilon)=\sum_{i=1}^{5} \lambda_{i} \varepsilon^{i} .
$$

The coefficients are chosen to be $\lambda_{1}=8.9615, \lambda_{2}=-27.1160, \lambda_{3}=28.8350, \lambda_{4}=-16.1349$ and $\lambda_{5}=6.4531$. These somehow arbitrary parameters can be obtained from a very simple micromechanical model as has been presented in [17]. The parameters for the corresponding trilinear approximation (7) (Illustrated in Fig. 2) are $k_{1}=k_{3}=3.76$ and $k_{2}=-1.76, \varepsilon_{b}=0.25$ and $\varepsilon_{h}=0.75$. From these values, the gradient stiffness $A=0.4669$ is calculated by numerically inverting (36) such that $\ell_{\text {neg }}=1$ which is related to the chosen cell size $s=1$.

\section{References}

1. Germain, P.: The method of virtual power in continuum mechanics. Part 2: Microstructure. SIAM J. Appl. Math. 25(3), 556-575 (1973)

2. Cosserat, E., Cosserat, F.: Deformable Bodies. 1. Auflage. Scientific Library A Hermann and Sons, Paris (1909)

3. Mindlin, R.D.: Second gradient of strain and surface-tension in linear elasticity. Int. J. Solids Struct. 1, 417-438 (1965)

4. Forest, S., Sievert, R.: Nonlinear microstrain theories. Int. J. Solids Struct. 43, 7224-7245 (2006)

5. Findeisen, C., Wackerfuß, J.: A general approximation of the exponential Cauchy-Born hypothesis to model arbitrarily shaped shell-like nanostructures within continuum mechanics. Int. J. Numer. Methods Eng. 105, 747-780 (2016)

6. Anderson, W.B., Lakes, R.S.: Size effects due to Cosserat elasticity and surface damage in closed-cell polymethacrylimide foam. J. Mater. Sci. 29, 6413-6419 (1994)

7. Eringen, A.C.: Linear theory of nonlocal elasticity and dispersion of plane waves. Int. J. Eng. Sci. 10(5), 425-435 (1972)

8. de Borst, R.: Simulation of strain localization: a reappraisal of the Cosserat continuum. Eng. Comput. 8, 317-332 (1991)

9. de Borst, R., et al.: Fundamental issues in finite element analysis of localization of deformation. Eng. Comput. 10, 99-121 (1993)

10. Forest, S., et al.: Continuum modeling of strain localization phenomena in metallic foams. J. Mater. Sci. 40, 5903-5910 (2005)

11. Mazière, M., Forest, S.: Strain gradient plasticity modeling and finite element simulation of Luders band formation and propagation. Contin. Mech. Thermodyn. 27, 83-104 (2015)

12. Antonakakis, T., Craster, R.V., Guenneau, S.: Homogenization for elastic photonic crystals and dynamic anisotropy. J. Mech. Phys. Solids 71, 84-96 (2014)

13. d'Agostino, M.V. et al.: Effective description of anisotropic wave dispersion in mechanical band-gap metamaterials via the relaxed micromorphic model. Appl. Phys. (preprint) (2018) 
14. Frenzel, T., Kadic, M., Wegener, M.: Three-dimensional mechanical meta-materials with a twist. Science 358, 1072-1074 (2017)

15. Seppecher, P., Alibert, J.-J., Dell'Isola, F.: Linear elastic trusses leading to continua with exotic mechanical interactions. J. Phys.: Conf. Ser. 319, 1-13 (2011)

16. Frenzel, T., et al.: Tailored buckling micro-lattices as reusable light-weight shock absorbers. Adv. Mater. 28, 5865-5870 (2016)

17. Findeisen, C., et al.: Characteristics of mechanical metamaterials based on buckling elements. J. Mech. Phys. Solids 102, 151-164 (2017)

18. Miehe, C.: Strain-driven homogenization of inelastic microstructures and composites based on an incremental variational formulation. Int. J. Numer. Methods Eng. 55, 1285-1322 (2002)

19. Forest, S., Trinh, D.: Generalized Continua and Non-homogeneous Boundary Conditions In Homogenisation Methods. Z. Angew. Math. Mech. 91(2), 90-109 (2011)

20. Jänicke, R., Steeb, H.: Minimal loading conditions for higher order numerical homogenisation schemes. Arch. Appl. Mech. 82(8), 10751088 (2009)

21. Hütter, G.: Homogenization of a Cauchy continuum towards a micromorphic continuum. J. Mech. Phys. Solids 99, 394-408 (2017)

22. Rokoš, O., et al.: Micromorphic computational homogenization for mechanical metamaterials with patterning fluctuation fields. J. Mech. Phys. Solids 123, 119-137 (2019)

23. Puglisi, G., Truskinovsky, L.: Mechanics of a discrete chain with bi-stable elements. J. Mech. Phys. Solids 48, 1-27 (2000)

24. Puglisi, G., Truskinovsky, L.: Rate independent hysteresis in a bi-stable chain. J. Mech. Phys. Solids 50, $165-187$ (2002)

25. Truskinovsky, L., Vainchtein, A.: The origin of nucleation peak in transformational plasticity. J. Mech. Phys. Solids 52, 1421-1446 (2004)

26. Triantafyllidis, N., Bardenhagen, S.: On higher order gradient continuum theories in 1-D nonlinear elasticity. Derivation from and comparison to the corresponding discrete models. J. Elast. 33, 259-293 (1993)

27. Forest, S.: Questioning size effects as predicted by strain gradient plasticity. J. Mech. Behav. Mater. 22, 101-110 (2003a)

28. Diebels, S., Steeb, H.: The size effect in foams and its theoretical and numerical investigation. Proc. R. Soc. Lond. A 458, $28692883(2002)$

29. Clerk-Maxwell, J.: On the dynamical evidence of the molecular constitution of bodies. Nature 11, 357-359 (1875)

30. Florijn, B., Coulais, C., van Hecke, M.: Programmable mechanical meta-materials. Phys. Rev. Lett. 113, 175503 (2014)

31. Shan, S., et al.: Multistable architected materials for trapping elastic strain energy. Adv. Mater. 27, 4296-4301 (2015)

32. Yang, H., Ma, L.: Multi-stable mechanical metamaterials by elastic buckling instability. J. Mater. Sci. 54, 3509-3526 (2019)

33. Le, K.: Introduction to Micromechanics: Classical and Quantum Mechanics, 1st edn. Nova Science, New York (2011)

34. Balk, A.M., Cherkaev, A.J., Slepyan, L.I.: Dynamics of chains with non-monotone stress-strain relations. I. Model and numerical experiments. J. Mech. Phys. Solids 49, 131-148 (2001)

35. Lebowitz, J.L., Penrose, O.: Rigorous treatment of the Van Der Waals-Maxwell theory of the liquid-vapor transition. J. Math. Phys. 7(1), 98-113 (1966)

36. Hallai, J., Kyriakides, S.: Underlying material response for Lüders-like instabilities. Int J. Plast. 47, 1-12 (2013)

37. Cottrell, A.H., Bilby, B.A.: Dislocation theory of yielding and strain ageing of iron. Proc. Phys. Soc. A 62, 49-62 (1949)

38. Johnston, W.G., Gilman, J.J.: Dislocation velocities, dislocation densities, and plastic flow in lithium fluoride crystals. J. Appl. Phys. 30(129), 129-144 (1959)

39. Shaw, J.A.H., Kyriakides, S.: On the nucleation and propagation of phase transformation in NiTi alloy. Acta Mater. 45, 683700 (1997)

40. Kyriakides, S., Miller, J.E.: On the propagation of Luders bands in steel strips. J. Appl. Mech. 67, 645-654 (2000)

41. Ameen, M.M., et al.: Size effects in nonlinear periodic materials exhibiting reversible pattern transformations. Mech. Mater. 124, 55-70 (2018)

42. Onck, P.R., Andrews, E.W., Gibson, L.J.: Size effects in ductile cellular solids. Part I: Modelling. Int. J. Mech. Sci. 43, 681-699 (2001)

43. Chen, C., Fleck, N.A.: Size effects in the constrained deformation of metallic foams. J. Mech. Phys. Solids 50, 955-977 (2002)

Publisher's Note Springer Nature remains neutral with regard to jurisdictional claims in published maps and institutional affiliations. 